\title{
EL AUTOR HA MUERTO. ¡LARGA VIDA AL AUTOR! ${ }^{1}$
}

\section{James C. MORRISON}

Boston College

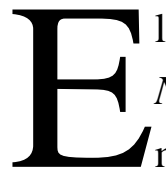
11 de junio de 1897, Mark Twain escribió la siguiente nota al corresponsal de Londres del New York Journal, como respuesta a una noticia reciente de aquel diario: «El anuncio de mi muerte fue una exageración»(Bartlett, 1992: 528). Si estuviera vivo hoy, supongo que Twain diría más de lo mismo con respecto a la afirmación postmodernista de la muerte del autor en general y su adopción por parte de los defensores del hipertexto. En Hypertext 2.0: The Convergence of Contemporary Critical Theory and Technology, George Landow (1997) -que, como sus libros, sigue con vida y buena salud- afirma que la tecnología del hipertexto manifiesta -0 , tomando prestada la jerga-, instancia, principios de la deconstrucción proclamados por Roland Barthes, Jacques Derrida, Michel Foucault, Mijaíl Bajtín, entre otros. Estas teorías, muchas de las cuales fueron desarrolladas teniendo en cuenta solamente textos estables e impresos, y antes incluso de que el software del hipertexto fuera ni siquiera creado, parecen converger con las de Vannevar Bush (1945) y Theodor Holm Nelson (1981 y 1987), el último de los cuales acuñó los términos de «hipertexto»e «hipermedia». Éstos encuentran encarnación, especialmente, en sistemas como Microcosm, Storyspace, DynaText, el ahora difunto Intermedia de la Universidad de Brown, y, hasta cierto punto, en la World Wide Web.

La afirmación concreta que concierne a este ensayo surge de la observación, hecha no sólo por los postmodernistas sino compartida también por I. A. Richards (1925 y 1929) y otros defensores modernistas de la Crítica Práctica, de que todos los textos son interpretados de formas varias, o «polisémicamente», por sus lectores. Los textos, por su misma naturaleza, están abiertos a interpretaciones diversas, y como resultado, no son en definitiva los textos escritos por sus autores, sino aquellos leídos por sus lectores. Los partidarios de la Nueva Crítica y del Postmodernismo discrepan en su actitud hacia esta rebeldía por parte del lector. En la Crítica Práctica -que se convirtió en la Nueva Crítica cuando fue transportada a través del Atlántico hacia el sur y hacia Yale-, el propósito de la crítica es el de aguzar las facultades perceptivas del lector y desengañarlo de nociones arbitrarias. En cambio, los postmodernistas celebran de hecho la sublevación del lector

\footnotetext{
${ }^{1}$ Este texto es una traducción de la conferencia inédita «The Author is Dead. Long Live the Author!», presentada en mayo de 2002 en el Massachusetts Institute of Technology (Cambridge). Lo reproducimos con la autorización del autor. La traducción es de Michelle Gama Leyva, con la colaboración de Aina Pérez Fontdevila.
} 
contra la autoridad ilegítima del autor hegemónico. He aquí cómo lo expone Barthes (1970) en $S / Z$, su estudio deconstructivo de la narración Sarrasine, de Honoré de Balzac:

Porque lo que está en juego en el trabajo literario (en la literatura como trabajo) es hacer del lector no ya un consumidor, sino un productor del texto. Nuestra literatura está marcada por el despiadado divorcio que la institución literaria mantiene entre el fabricante y el usuario del texto, su propietario y su cliente, su autor y su lector. Este lector está sumergido en una especie de ocio, de intransitividad, y, ¿por qué no decirlo?, de seriedad: en lugar de jugar él mismo, de acceder plenamente al encantamiento del significante, a la voluptuosidad de la escritura, no le queda más que la pobre libertad de recibir o rechazar el texto: la lectura no es más que un referéndum. Por lo tanto, frente al texto escribible se establece su contravalor, su valor negativo, reactivo: lo que puede ser leído pero no escrito: lo legible. Llamaremos clásico a todo texto legible. $(2004: 2)^{2}$

Por lo tanto, en el texto postmoderno encontramos la «muerte del autor», que tiene lugar porque el escritor -y ya no el autor- le cede al lector, en alguna medida, el control sobre el punto de entrada, el orden y el modo de proceder a través del texto. Me gustaría examinar cómo Landow y otros, como Jay David Bolter (1991 y 2001), aplican la teoría crítica a la comprensión de las implicaciones del hipertexto para el futuro del libro. Quisiera sugerir que, cuando se los enfrenta a algunas de las realidades editoriales en la historia, en la actualidad, y a las que probablemente existan en un futuro cercano, las afirmaciones acerca de la naturaleza transformativa del hipertexto son, por lo menos, exageradas, y los dictámenes sobre la muerte del autor, por las mismas razones, prematuros.

En Hypertext 2.0, Landow (1997) muestra cómo los sistemas hipertextuales conceden tanto a los creadores como a los usuarios de redes hipertextuales diversos grados de agencia sobre la generación y el uso de los hipervínculos. Por ejemplo:

1. En Storyspace, el texto consiste en unidades de lectura cortas y adaptadas al tamaño de la pantalla, también llamadas lexías (usando un término acuñado por Barthes). El autor crea una red de asociaciones entre las lexías a partir de cualquier principio que se adapte al tema o al género, ya sean repeticiones de la misma palabra o frase, términos clave, temas o materias. El/la autor/a también puede proporcionar al lector diversas interfaces para el texto, así como recursos de navegación como hojas de ruta [roadmap], lectores de página [page reader] o visores storyspace, lo que proporciona al lector la posibilidad de elegir diferentes vías para recorrer las lexías.

2. En Microcosm, el autor crea una red de conexiones entre documentos o archivos en cualquier soporte, designando cada archivo con palabras o frases clave que describen su tema o materia. El documento o archivo multimedia es solicitado cada vez que el lector activa la frase o palabra clave en otro archivo, que puede solicitar otros muchos documentos o archivos relacionados. Estos vínculos, llamados genéricos o implícitos, sólo surgen si son requeridos por el lector. Ningún formato de texto especial ni ninguna clase de botón indica la presencia de material hipervinculado, sino que los hipervínculos sólo son activados por un lector que adopte un rol activo en relación con el texto, en el sentido que tiene que clicar el texto para que se revelen las conexiones. Los usuarios también pueden añadir materiales en cualquier aplicación que se ejecute en el sistema operativo

\footnotetext{
${ }^{2}$ Usamos la traducción de Nicolás Rosa.
} 
subyacente y hacer que esos archivos sean accesibles diseñando hilos de texto que activan los hipervínculos genéricos.

3. En Intermedia, de la Universidad de Brown, los usuarios pueden solicitar un tema de red que consiste en una variedad de materiales temáticos relacionados y comentarios adjuntos, hipervinculados a textos propios y a materiales externos relacionados con el tema. También proporciona a los usurarios diversos recursos de orientación gráfica.

4. En Xanadu, imaginada por Ted Nelson, los usuarios podrían usar y publicar libremente material disponible en abierto a través de lo que él llama Docuverse, con un sistema de micropagos para gestionar el problema de los derechos de reproducción de forma análoga al copyright en el mundo impreso.

Queda claro, con estos ejemplos, que el hipertexto ofrece a los lectores un conjunto de controles muy diferente en la experiencia de lectura. De hecho, leer sobre una pantalla es un tipo de experiencia paradigmáticamente diferente a leer una página impresa, análoga, en algunos sentidos, a moverse del asiento del pasajero al del conductor en un automóvil. Pero la cuestión que emerge es: ¿por qué dotar al lector de un mayor sentido de agencia disminuye necesariamente la agencia del autor? ¿Por qué tal situación debería ser presentada siempre como un juego en el que uno gana y otro pierde?

\section{Una paradoja}

Una pregunta aún más esencial es: ¿por qué, si el autor está muerto, hay tantos autores escribiendo sobre lo muerto que está el autor? ¿Estos autores proponen seriamente que las palabras de cualquier lector, a todos los efectos, son igual de válidas? Si lo son, esto convierte en igual de válido que uno diga que el/la autor/a no está muerto/a como que diga que lo está. O que la tecnología informática, para usar de nuevo la jerga, no instancia las teorías postmodernistas como que sí lo hace. Las afirmaciones de la muerte del autor por parte de autores parecen resumir las condiciones de la paradoja de Epiménides: «Todos los cretenses son mentirosos... Uno de sus propios poetas lo ha dicho» (Weisstein, 1999a). Una analogía incluso más mordaz sería la paradoja de Eubulides: «Esta afirmación es falsa» (Weisstein, 1999b). Si es cierta, entonces tiene que ser falsa, pero si es falsa, entonces debe ser verdadera. Uno también recuerda las ironías cruzadas de aquel título de Abbie Hoffman (1970), aparentemente inmortal: Roba este libro [Steal this book].

La ironía se vuelve aún más exquisita por el hecho de que, en 1995, Roba este libro fue publicado en una edición para bibliotecas y en otra facsímil con motivo de su veinticinco aniversario, y en la contraportada de la reedición más reciente el editor anunciaba de nuevo: «Esta edición de tapa blanda de Roba este libro es la edición original, la ÚNICA autorizada por el mismo Abbie (antes de morir). Todas los regalías provenientes de las ventas de este libro irán a la Fundación Activista Abbie Hoffman, dirigida por sus herederos».

Ciertamente, los sistemas hipertextuales como la Intermedia de la Universidad de Brown (ahora difunta) y Microcosm, permiten a los lectores agregar notas a un texto, proporcionar 
hipervínculos a materiales relacionados y compartir visualizaciones de manera colaborativa, todo lo cual puede ser solicitado por cualquier otro usuario. Estas son útiles funciones en cualquier sistema de aprendizaje, pero sugerir que esta expansión de las posibilidades a la hora de compartir, por parte de cualquiera, sus propias anotaciones, constituye la negación del autor original o desdibuja la distinción entre el autor y los lectores, parece reclamar un fundamento mucho más concreto del que se le ha dado. El mismo Landow señala que la copia del archivo original solicitado permanece intacta respecto a los cambios o adiciones realizadas por cualquiera que no sea su autor original (Landow, 1997: 90). La «virtualidad» del texto parece entonces no tener nada que ver con el control autorial sobre una obra original.

Landow continúa argumentando:

pero esto sí reduce la distancia fenomenológica que separa los documentos individuales entre ellos en los mundos de la impresión y del manuscrito. Reduciendo la autonomía del texto, el hipertexto reduce la autonomía del autor. En palabras de Michael Heim [en Electric Language], «como el carácter autoritario del texto disminuye, también lo hace el reconocimiento del yo privado del autor creativo». [...] El hipertexto y la teoría contemporánea reconciben el autor en un segundo modo. Como podemos observar cuando examinamos la noción de escritura colaborativa, ambos están de acuerdo en configurar al autor del texto como un texto. Como explica Barthes en su famosa exposición de esta idea, "este "yo" que se aproxima al texto ya es en sí mismo una pluralidad de otros textos, códigos que son infinitos». (Landow, 1997: 90-91)

Si vinculamos esto a la idea deconstructiva según la cual todos los textos son simplemente redes de significantes sin ninguna conexión necesaria con un significado, el autor es despojado de existencia, convirtiéndose en una mera ficción conveniente que leemos en el texto para volverlo más cómodo y legible. Para poner a prueba esta cuestión, examinemos la evidencia que Landow expone para fundamentarla: su discusión sobre la escritura colaborativa tanto en entornos impresos como hipertextuales.

Para empezar, Landow plantea una marcada distinción entre las prácticas y las actitudes hacia la autoría en la ciencia y en las humanidades. En la ciencia, la autoría compartida de investigaciones publicadas se da por sentada, ya que la investigación de estudiantes licenciados está generalmente apoyada mediante el financiamiento de grupos de laboratorio bajo la supervisión de un director como supervisor. En el caso de las humanidades, en cambio, la investigación de los estudiantes está financiada, en su mayor parte, por becas de docencia y, aunque los supervisores llevan a cabo funciones análogas a sus homólogos científicos, el asesor nunca es incluido como co-autor (Landow, 1997: 106-107). Por lo tanto, «una de las razones de las diferentes concepciones de la autoría y de la propiedad intelectual en las humanidades y en las ciencias reside en las diferentes condiciones de financiación y las diferentes políticas resultantes para cada disciplina» (Landow, 1997: 107).

Pero, entonces, va más allá de esta explicación social constructivista de tales diferencias hasta acusar a las humanidades de distorsionar la verdadera naturaleza de la autoría:

Otro corolario es que las disciplinas humanísticas, que tradicionalmente aplican enfoques históricos a sus áreas de estudio, consideran sus propias suposiciones sobre la autoría, la propiedad autorial, la creatividad y la originalidad como verdades eternas. En particular, los estudios literarios y las instituciones literarias, como los departamentos de inglés - que todavía nadan en el resplandor del Romanticismo-, 
exageran acríticamente las nociones románticas de creatividad y originalidad hasta lo absurdo. (Landow, 1997: 107)

Se puede detectar un deje de amargura en esta observación, que puede atribuirse a las tensiones experimentadas por alguien cuyas actividades han cruzado las fronteras de las humanidades y de las ciencias informáticas tan decididamente. Pero como afirmación reveladora de la causa raíz del asunto, está en sí misma envuelta en una distorsión. Uno se pregunta si él extendería esta caricatura de las humanidades a los autores que han proporcionado el fundamento teórico de su propio trabajo Barthes, Derrida, Foucault y Bajtín, entre otros-. ¿La validez de sus teorías adolece de exageradas «nociones románticas de creatividad y originalidad»? Si es así, volvemos al enigma de la paradoja del mentiroso. Pero si no, tiene que haber alguna otra explicación para la tendencia humanística a no considerar el trabajo colaborativo como «original» y a que éste no tenga peso en cuestiones tales como el acceso o la promoción en la academia.

Dejando a un lado cuestiones de financiación y política, está claro que, en la actualidad, la colaboración en las ciencias empíricas y teóricas no es solamente una condición deseable sino necesaria para generar nuevo conocimiento. El tamaño y la complejidad de la investigación científica en sociedades tecnológicamente avanzadas hacen ineludibles estas condiciones de trabajo en equipo. Pero no siempre fue así. Los principios matemáticos y científicos en los que se basa la investigación actual fueron desarrollados por filósofos naturales, y autoriales, como Copérnico, Newton, Leibniz y Descartes, cuyos descubrimientos no emanaron del equipo de un laboratorio financiado, sino de la mente de un individuo. Como apuntan Elizabeth Eisenstein (1979) y Marshall McLuhan (1962), fue la imprenta la que hizo posible que avances de esta envergadura tuvieran lugar en la ciencia empírica a un paso tan acelerado. Sin embargo, observando la misma evidencia, Landow parece que sólo quiere enfatizar los aspectos negativos, afirmando que:

la tecnología del libro y las actitudes que sostiene son las instituciones en mayor medida responsables del mantenimiento de las nociones exageradas de individualidad autorial, singularidad y propiedad que tantas veces falsifican drásticamente la concepción de las contribuciones originales en las humanidades y transmiten imágenes distorsionadas de la investigación. (Landow, 1997: 108)

\section{La imprenta como paradigma}

Hay reconocer, por supuesto, que Landow no carece de razón en este punto. Como observa Eisenstein:

La cultura escribana [scribal culture] no pudo soportar la patente de invenciones o los derechos de copia de composiciones literarias. Funcionó en contra del concepto de los derechos de propiedad individual. No se prestó a la preservación de los trazos de las idiosincrasias personales, a la difusión pública de los pensamientos privados, ni a ninguna de las formas de publicidad silenciosa que han moldeado la consciencia del individuo durante los últimos cinco siglos. (Eisenstein, 1979: 229-230)

Por otra parte, «el culto a la personalidad fue repetidamente socavado por las condiciones de la cultura escribana y fue poderosamente reforzado tras el advenimiento de la imprenta» (Eisenstein, 1979: 232). Aun así, enfatizar este aspecto de la cultura de la imprenta es excluir otras evidencias 
aportadas por Eisenstein, entre otros, según las cuales la imprenta y la industria editorial proporcionaron oportunidades, por primera vez, para el trabajo colaborativo en la producción de trabajos impresos que la cultura escribana no podía sostener.

Dejando a un lado la labor añadida de los rubricadores y los iluminadores en la producción de manuscritos, el trabajo de un escriba era esencialmente una ocupación solitaria, con un solo hombre produciendo una sola copia de un trabajo cada vez. Con el incremento de la demanda de libros tras el crecimiento de las universidades medievales, el proceso fue aumentado con lo que era conocido como «sistema de la pecia», en el cual se separaban cuadernos de libros y eran prestados individualmente para ser copiados por los mismos estudiantes o por copistas contratados (Eisenstein, 1979: 12-14). La invención de la imprenta impulsó por primera vez el desarrollo de un proceso en cadena, en el que era necesaria la colaboración de editores, compositores, impresores, correctores de estilo, operarios, aprendices, linotipistas y libreros, frecuentemente bajo el mismo techo. Dada la considerable inversión de capital requerida para este tipo de producciones, la cooperación entre prestamistas y comerciantes viajeros era indispensable, estableciendo así muchas de las bases del capitalismo y la mercadotecnia modernos, característicos de la industria editorial, además de otras tantas iniciativas propias de la Edad Moderna (Eisenstein, 1979: 3-71). Así, la tecnología del libro fue responsable del establecimiento no sólo de las nociones de autoría y de derechos de autor que Landow nos acusa de naturalizar, sino también de las mismas condiciones de colaboración necesarias para producir cualquier tipo de obra publicada, ya sea impresa o hipertextual.

Hay que reconocer que las mismas tecnologías que producen hipertextos fueron posibles solamente gracias al cúmulo de libros y artículos impresos que respaldan su conceptualización, desarrollo y documentación. Estos trabajos impresos son producto no sólo de otros textos, sino de autores reales cuya presencia en la obra es vital para su concepción, producción y distribución. Pero tales obras sólo pueden ser publicadas a través de los esfuerzos cooperativos y colaborativos de un gran número de personas cuyos trabajos y roles han sido establecidos a lo largo de quinientos años de cultura de la imprenta. Cuando se crean hipertextos, puede que la naturaleza y los nombres de esos trabajos se modifiquen, pero sus funciones, mutatis mutandis, no desaparecen. Por ello, es un poco desconcertante que nuestro autor (Landow) ofrezca una explicación de la producción de su Dicken's Web para el sistema Intermedia (1997: 111-114) en los términos en que lo hace, como un nuevo paradigma de publicación y como un ejemplo del modo en que la producción hipertextual desdibuja la identidad del autor: «Crear la Dicken's Web involucró a docenas de "autores" y al menos otros tantos tipos de colaboración» (1997: 111).

Hay demasiados detalles en los que se podría profundizar aquí, pero, esencialmente, lo que Landow describe es un proceso que no es significativamente diferente al que atraviesa un editor cuando crea un reader, una antología, una edición crítica, un libro de referencias, o cualquier otro tipo de compendio enciclopédico impreso. Existe un editor general -no un editor de manuscritos o de adquisiciones, sino un «nombre» con credenciales académicas- que concibe el proyecto, proporciona el contenido o, por lo menos, parte de éste, y organiza el proceso de reunión de los textos de otros 
colaboradores: a él se otorga el papel de «autor». Ante cualquier necesidad visual, existe un editor de arte que involucra a artistas propios o independientes para satisfacerla. Otras personas pueden ser invitadas a contribuir con materiales suplementarios en forma de trabajos por encargo. La obtención de permisos para el uso de contenidos de terceros es responsabilidad del autor, de los colaboradores individuales o de un gestor de derechos de la editorial. Aquellos que contribuyeron con textos originales o con descripciones del trabajo de otros son reconocidos en los créditos correspondientes, mientras que en una obra enciclopédica los colaboradores son generalmente identificados por sus iniciales al final de cada pieza individual. Ningún aspecto de esta descripción del proceso editorial es incompatible con la que ofrece Landow en relación a la creación de The Dickens Web, aunque él lo presente como un «complejo problema de autoría» (1997: 113) y lo resuma afirmando: «Como deja claro esta explicación, la "autoría" de textos individuales en un entorno hipermediático se vuelve aún más problemática que en el mundo de la imprenta» (1997: 114). Pero uno busca en vano razones convincentes para considerarlo así.

Si sabemos que Samuel Johnson fue el autor de su Dictionary of the English Language, y que Noah Webster escribió An American Dictionary of the English Language, ¿quién es el autor del Webster's Third New International Dictionary, Unabridged? ¿Quién es el autor de la Enciclopedia Británica? ¿O de The Cambridge Chaucer Companion, o de Our Exagmination Round His Factification For Incamination of Work in Progress, o del Chilton's Repair and Tune-Up Guide, Capri, 1970-1977, o de The Holy Bible, King James Version?

De estos ejemplos se colige que la noción de autoría compartida o indeterminada, en todas estas obras creadas en la cultura de la imprenta, no necesita el hipertexto. En esta misma línea, los protohipertextos, y muchos de los hipertextos que han sido publicados, tienen autores claramente definidos, cuyas obras son buscadas mayoritariamente por la fuerza de la reputación y del prestigio de sus autores. Quien lee Rayuela, de Julio Cortazar; Glas, de Jacques Derrida; o S/Z, de Roland Barthes, lo hace sin duda porque quiere leer obras de esos autores. Y, sin importar cuánto control les cedan o no a sus lectores, Michael Joyce, Stuart Moulthrop y Shelley Jackson siempre serán claramente identificados como los autores de Afternoon, Victory Garden y Patchwork Girl, respectivamente.

Por otra parte, no hay ninguna evidencia que sugiera que la autoría está en peligro de desaparecer en el campo de la edición de libros, inmensamente más grande. De hecho, hay evidencias considerables de lo contrario. En Book Business, Jason Epstein apunta que:

Entre 1986 y 1996, [...] sesenta y tres de los cien títulos más vendidos fueron escritos por tan sólo seis escritores: Tom Clancy, John Grisham, Stephen King, Dean Koontz, Michael Crichton y Danielle Steel - una concentración mucho mayor que en el pasado, y una moneda de dos caras para los editores, quienes sacrifican una gran parte de su beneficio habitual, y a menudo sufren pérdidas, para mantener autores influyentes como éstos-. Pero los nombres de marca de autores superventas pueden seguir la innovación de King [publicando Riding the Bullet en la Web como descarga] como un siguiente paso lógico y explotar sus derechos electrónicos sin la ayuda de sus editores. (Epstein, 2001: 33-34) 
Si esta es una tendencia significativa y duradera, podría parecer que, en gran medida, la publicación electrónica no sólo está favoreciendo la continuación del texto legible, sino que, al hacerlo, fortalece la figura del autor hegemónico. Bajo estas condiciones, tendría sentido preocuparse más por la muerte de la editorial que por la del autor. De manera similar, las dificultades recientes de Doris Kearns Goodwin y de Stephen Ambrose por haber sido acusados de plagiar las obras de Lynne McTaggart y de Thomas Childers, respectivamente, demuestran que los derechos de propiedad de los autores están lejos de haberse erosionado, y que incluso están entrando en una etapa de defensa aún más vigorosa. Ciertamente, la aprobación de la Digital Millennium Copyright Act y los intentos de la industria musical para proteger sus derechos a la luz de amenazas como las de Napster, muestran que la digitalización de la propiedad intelectual sólo ha aumentado las presiones para la defensa de los derechos de autor. El hecho de que este movimiento se lleve a cabo en nombre del autor o del editor es ajeno a la cuestión, esto es, saber si los desafíos de la publicación electrónica a los propietarios de derechos de autor tradicionales y arraigados van justamente en dirección opuesta a la de las afirmaciones postmodernistas.

\section{Recuperar el manuscrito}

¿Por qué los defensores del hipertexto creen tan firmemente en la muerte del autor? Una posible respuesta es sugerida en el análisis del hipertexto de Stuart Moulthrop (1991), que utiliza la tétrada de McLuhan, desarrollada en «Las leyes de los medios» (McLuhan y McLuhan, 2009). El análisis de Moulthrop es lúcido, al darse cuenta de que:

\footnotetext{
El [h]ipertexto difiere de medios de comunicación anteriores en que no es algo nuevo en absoluto sino un retorno o un regreso [...] a una forma más temprana del discurso simbólico, esto es, la imprenta [...]. En el núcleo del concepto de hipertexto residen ideas de afiliación, correspondencia y resonancia. En esto, como [Ted] Nelson ha sostenido desde el principio, el hipertexto no es nada más que la extensión de lo que la literatura ha sido siempre (al menos desde «La tradición y el talento individual» [de T. S. Eliot]) una red de relaciones temporalmente extendida en la que sucesivas generaciones de lectores y escritores hacen y deshacen perpetuamente-. (1991: 18-19)
}

Los deconstruccionistas tienen razón cuando afirman que los textos siempre han estado abiertos y que la tecnología específica de la imprenta fue la responsable del fomento de la noción de texto unitario. Pero los lectores siempre han reconocido que los autores son capaces de escribir libros solamente porque han leído otros libros y los utilizan para sus propios propósitos. Como ya dijo T. S. Eliot, de modo célebre, en su ensayo sobre Philip Massinger: «Los poetas inmaduros imitan, los poetas maduros roban... » (1975: 153).

En vez de considerarlo la muerte del autor, sería más útil ver que los recursos que tienen a disposición los usuarios de hipertextos les permiten crear manuscritos electrónicos iluminados por sî mismos, ampliando la definición de autoría al recuperar la definición medieval. Como he sugerido en otras ocasiones (Morrison, 2000), Landow afirma [que] «[1] os manuscritos medievales presentan algún tipo de combinación hipertextual de tamaños de letra, anotaciones al margen, ilustraciones y adornos visuales, tanto en forma de caligrafía como de adiciones pictóricas» (1997: 63). Este es un 
enfoque prometedor, porque parece reconocer que la comunicación electrónica a través del ordenador está retomando, con variaciones, muchas de las condiciones de la producción y consumo del manuscrito medieval. También sugiere conexiones entre la pantalla del ordenador y el concepto de «luz al través» de vitrales y manuscritos iluminados, como propone McLuhan (1962: 105-109).

En el siglo XIII, San Buenaventura escribió que existen cuatro roles que puede asumir alguien que hace libros:

Un hombre puede escribir las obras de otros, sin agregar o cambiar nada, en cuyo caso será simplemente llamado «escriba» (scriptor). Otro escribe las obras de otros con añadidos que no le son propios; y es llamado «compilador» (compilator). Otro escribe la obra de otros y la suya propia, pero con la obra de otros en lugar principal, añadiendo la suya con propósitos explicativos; y es llamado «comentarista» (commentator). [...] Otro escribe tanto su propia obra como la obra de otros, pero con la suya en lugar principal, añadiendo la de otros con propósitos de confirmación; este hombre puede ser llamado «autor» (auctor). (Eisenstein, 1979: 121-122)

Se pueden observar fuertes similitudes entre estos «fabricantes de libros» y la concepción de Landow del autor hipertextual posmoderno. En ambos casos, la autoría es una cuestión de grado más que un rol claramente definido y, en ningún caso, la creación original ex nihilo es parte del cuadro. $\mathrm{El}$ «fabricante de libros» medieval no podía concentrarse en crear composiciones originales porque muchas de sus energías estaban necesariamente enfocadas en preservar la escritura del pasado. La situación no era la de hoy, en «la era tardía de la imprenta»(Bolter, 2001: 2-3), cuando uno está obligado a citar y a dar crédito a autores anteriores cuya obra está frecuentemente disponible. Esta última condición es, por supuesto, producto de la mercantilización de libros que posibilitó la invención de la imprenta y de la tradición de derechos de autor que le siguió gradualmente. El «fabricante de libros» medieval tenía una obligación principal bastante diferente, que consistía en asegurarse, en primer lugar, de que las obras con las que trabajaba fueran preservadas, ya que para ello no existían más medios que sus dedos y sus manos. Las energías que quedaran después de cumplir este acto de preservación, eran, en cierto modo, un suplemento, y no el objetivo primordial de su energía.

Por supuesto, en la actualidad los trabajadores del conocimiento no tenemos que preocuparnos por estos asuntos - de hecho, nuestro problema es justamente el contrario-. Como muchos comentaristas nos recuerdan, nuestro problema no es tanto preservar textos escasos como desgranar la paja de lo que hoy llamamos «información» hasta darle sentido a nuestro mundo. Como expone Neil Postman (1990), corremos el peligro de «informarnos hasta la muerte». En la «era industrial de la imprenta» (Bolter, 2001: 2), un período bastante breve entre la Revolución francesa y la Primera Guerra Mundial -que ahora parece haber sido una etapa de gentileza eduardiana relativamente relajada-, la autoría no era una experiencia tan angustiosa. Los autores podían dedicar sus energías a desarrollar una voz consistente y unificada, un punto de vista, retomando la metáfora de McLuhan, del cual podría emanar el mundo de su libro. Pero con el desarrollo de la imprenta de vapor y el crecimiento de la materia impresa producida, el mundo de la impresión se expandió hasta la sobrecarga, dando como resultado la fragmentación y la incoherencia, contra las que reaccionaron 
los manifiestos y las manifestaciones del Futurismo, el Dadaísmo y el Constructivismo, creando, en otro término de McLuhan, un antiambiente. Con la posterior explosión de información posibilitada por el almacenamiento electrónico, de la cual el memex de Vannevar Bush (1945) fue el primer antiambiente conceptualizado, se hizo necesario lidiar con la información no sólo a un nivel superficial sino también en lo que Stuart Moulthrop llama su

«forma» hipotextual - por ejemplo, el modo en que los nódulos están divididos para dar cabida a estructuras de datos y a estrategias de visualización, o los tipos de vinculación disponibles y las formas en que son evidentes para el lector-. En términos prácticos, esto significa que se espera que los usuarios de un sistema hipertextual entiendan la impresión no sólo como el medio del discurso literario tradicional, sino también como una metaherramienta, la clave para el poder con respecto al propio sistema. (1991: 35)

Por consiguiente, la presión sobre el creador contemporáneo de hipertextos no se dirige a la preservación, como ocurría con el fabricante de libros medieval, sino a la programación de la interfaz o al trabajo con una interfaz previamente programada. Las energías mentales invertidas en la manipulación de la información en el sistema sitúan estas actividades en el campo de lo que Moulthrop llama «alfabetización secundaria»:

Ong y McLuhan han sostenido que la televisión y la radio introducen una «oralidad secundaria», un retorno a las formas no impresas del lenguaje y a un «espacio auditivo» de cognición [Oralidad y escritura; «Leyes de los medios»). Por analogía, el hipertexto y la hipermedia parece probable que instiguen a una alfabetización secundaria -«secundaria» en tanto que esta aproximación a la lectura y a la escritura implica una autoconsciencia sobre la mediación tecnológica de estos actos, una sensibilidad hacia el modo en que los textos-bajo-el-texto constituyen otro orden detrás de lo visible-. Esta alfabetización secundaria conlleva tanto la retórica como la técnica: leer en el nivel hipotextual es confrontar (de modo paragnóstico) el diseño del sistema; escribir en este nivel es reprogramar, revisando el trabajo del primer fabricante. Por consiguiente, esta alfabetización secundaria abre a sus lectores un «ciberespacio» en el verdadero sentido de la palabra, es decir, un lugar de mando y control donde la palabra escrita tiene el poder de rehacer las apariencias. (1991: 36)

La «alfabetización secundaria» de Moulthrop tiene características comunes con el concepto de «alfabetización artesanal» de Eric A. Havelock, esto es, una «alfabetización de un tipo secundario» (1986: 41), «una pericia que sólo posee un restringido grupo de la población» (1982: 188). Este es el nivel de alfabetización que prevalece en toda sociedad en la que los medios de producción de textos están limitados a un pequeño grupo o clase porque (a) los materiales de producción son resistentes y requieren años de aprendizaje antes de que puedan ser completamente dominados, y porque (b) el código de la lengua hablada tiene un sistema simbólico tan ambiguo o tan complejo que la responsabilidad de generar el texto tiene que ser confiada a una élite de escribas especialmente versados en dicho código a través de años de escolarización y de estudios teóricos. Estas condiciones se han mantenido en todas las culturas en las que el alfabeto fonético establecido por los antiguos griegos a partir del sistema de consonantes de los fenicios no ha sido plenamente adoptado e internalizado por el grueso de la población - esto es, culturas no-Occidentales, la cultura griega hasta al menos el siglo IV a. C. y la cultura europea del Medioevo-. Son justamente estas condiciones las que se están repitiendo a causa del requisito añadido del hipertexto: que creadores y usuarios dominen la manipulación del texto a un nivel de sistema «hipotextual». Recuperando el manuscrito 
iluminado, se podría decir que el hipertexto y los sistemas hipermediáticos están recreando el ethos no democrático, o incluso antidemocrático, de la alfabetización artesanal de la Edad Media. Por lo tanto, es de esperar que el hipertexto no haga mucho por borrar la «brecha digital», sino que, de hecho, la aumente, en connivencia, particularmente, con los efectos negativos del extendido consumo de televisión sobre los niveles de alfabetización.

Pese al supuesto incremento del grado de democracia que se otorga a los usuarios del hipertexto a la hora de controlar sus interacciones con lo que leen, la carga añadida al tener que aprender a usar el sistema operativo metatextual, ya sea como escritor o como lector $-\mathrm{o}$ como ambos-, interpone en realidad una serie de obstáculos a la lectura que son indudablemente no democráticos. Debemos recordar que los centros de formación en hipertexto son instituciones de élite tales como el Massachusetts Institute of Technology, la Universidad de Brown, el Darthmouth College, el Georgia Institute of Technology y la Universidad de Carnegie Mellon, y que los modelos teóricos del movimiento tienen su origen en el Collège de France y en la École Normale Supérieure. Independientemente de lo inclusivas y meritocráticas que aspiren a ser algunas de estas instituciones, son inevitablemente deficientes a la hora de inculcar el hipertexto en la cultura en general, debido a la simple lógica de los números. Michael Joyce (1988: 14) ha afirmado que la hipermedia es «la venganza del texto contra la televisión» (Bolter, 1991: 26), pero uno tendría que ser un pedante empedernido para no reconocer que Oprah Winfrey ha hecho más para promover la literatura de lo que cualquier promotor del hipertexto ha hecho o probablemente hará. Hay que decir que los deconstruccionistas probablemente sí lo reconocerían, aunque luego añadirían que esta situación no es la mejor. Dirían que todo lo que ella ha estado haciendo es promover textos legibles, que se contentan con permitir a los lectores regodearse con las cadenas de su servidumbre a una autoría al estilo de Simon Legree. Mejor tomar el camino de Nat Turner -dirían los deconstuccionistas- y liberarse de la servidumbre del autor como amo, sin importar las consecuencias.

En La galaxia Gutenberg, Marshall McLuhan declaró:

Vivimos hoy en el primer período de una era para la que el significado de la cultura de la imprenta se está haciendo tan extraño como el significado de la cultura del manuscrito lo fue para el siglo XVIII. [...] Lejos de querer achicar la cultura mecánica de la época de Gutenberg, estimo que debemos ahora esforzarnos mucho por conservar sus logrados valores. (1969: 135)

Creo que a esto se resume el ataque al autor. El desafío postmodernista a la cultura de la imprenta no se dirige esencialmente a su técnica, sino a su ethos. Las declaraciones sobre la muerte del autor no son solamente exageraciones, sino más bien las últimas bocanadas de la filosofía de la «Literatura del Agotamiento» (Barth, 1967: 29-34), cultivada en el invernadero de la academia y a la que se le ha permitido prolongarse cuando ya debería haber caído por su propio peso. La orquídea de la decadencia, esta fleur du mal, persiste en un entorno artificial que con el tiempo se esfumará, provocando su deceso. Mientras tanto, la flor silvestre del libre pensamiento, esta pensée sauvage, siempre adaptándose al cambio, prospera al aire libre. 
Si el autor posmoderno está muerto, como consecuencia de fuerzas naturales, entonces, como verdaderos sujetos, sólo nos queda recurrir a la continuidad de la autoría que el poder de la imprenta ha proporcionado a la esfera cultural y proclamar: « $\mathrm{E}$ El autor ha muerto; larga vida al autor!».

\section{Referencias bibliográficas}

BARTH, J. (1967): «The literature of exhaustion», The Atlantic, 220, 2.

BARTHES, R. (1971): S/Z. Trad. de Nicolás Rosa. Buenos Aires, Siglo XXI, 2004.

BARTLETT, J. (1992): Familiar quotations: A collection of passages, phrases, and proverbs traced to their sources in ancient and modern literatura. Boston, Little / Brown.

BOLTER, J. D. (1991): Writing space: The computer, hypertext, and the history of writing. Hillsdale, Erlbaum.

- (2001): Writing space: Computers, hypertext, and the remediation of print. Mahwah, Erlbaum.

Bush, V. (1945): «As we may think», The Atlantic Monthly, 176/1, en http://www.theatlantic.com/ unbound/flashbks/computer/bushf.htm.

EISENSTEIN, E. L. (1979): The printing press as an agent of change. Cambridge, Cambridge University Press. [La imprenta como agente de cambio. Trad. de K. Bello, México, FCE, 2010]

Eliot, T. S. (1975): Selected prose of T. S. Eliot. Nueva York, Harcourt Brace Jovanovich and Farrar, Straus and Giroux.

EPSTEIN, J. (2001): Book business: Publishing past present and future. Nueva York, Norton.

HAVELOCK, E. A. (1982): The literate revolution in Greece and its cultural consequences. Princeton, Princeton UP.

- (1986): La musa aprende a escribir. Reflexiones sobre oralidad y escritura desde la Antigüedad hasta el presente. Trad. de Luis Bredlow Wenda. Barcelona, Paidós, 1996.

HofFMAN, A. (1970): Steal this book. Nueva York, Four Walls Eight Windows, 2002.

JoYCE, M. (1988): «Siren shapes: Exploratory and constructive hypertexts», Academic Computing 3 (4), pp. 10-14, 37-42.

LANDOW, G. (1997): Hypertext 2.0: The convergence of contemporary critical theory and technology. Baltimore, Johns Hopkins UP.

McLuhan, M. (1962): The Gutenberg galaxy: The making of typographic man. Toronto, University of Toronto Press. [La galaxia Gutenberg: génesis del «homo typographicus». Madrid, Aguilar, 1969]

- y McLuhan, E. (1988): «Las leyes de los medios». Trad. de Eva Aladro Vico. Cuadernos de Información y Comunicación, 14, 2009, pp. 285-316.

MorRison, J. C. (2000): «Hypermedia and synesthesia», Media Ecology Association, en http://www. mediaecology.org/publications/MEA proceedings/v1/hypermedia and synesthesia.html.

Moulthrop, S. (1991): «You say you want a revolution? Hypertext and the laws of media», Postmodern Culture, 1/3, en http://muse.jhu.edu/journals/postmodern culture/v001/1.3moulthrop.html.

Nelson, T. H. (1981): Literary machines. Swarthmore, publicación del autor. 
- (1987): Computer lib/dream machines. Seattle, Microsoft Press.

Postman, N. (1990): «Informing ourselves to death», comunicación en el encuentro de la German Informatics Society (Gesellschaft für Informatik), Stuttgart.

RichaRdS, I. A. (1925): Principles of literary criticism, Nueva York, Harcourt, Brace \& World.

— (1929): Practical criticism. Nueva York, Harcourt, Brace \& World. [Crítica Práctica. Trad. de H. Valentí. Madrid, Visor, 1991]

WeISSTEIN, E. W. (1999a): «Epimenides paradox», en http://mathworld.wolfram.com/Epimenides Paradox.html.

(1999b): «Eubulides paradox», en http://mathworld.wolfram.com/EubulidesParadox.html. 